\title{
Características obstétricas das gestantes submetidas à cesariana segundo a Classificação de Robson
}

\author{
Obstetric characteristics of pregnant women undergoing cesarean section, by the Robson Classification \\ Características obstétricas de las mujeres embarazadas sometidas a cesárea según la Clasificación \\ de Robson
}

Liendne Penha Abreu'; Rivaldo Lira Filho"; Roseane Lustosa de Santana'll

\begin{abstract}
RESUMO
Objetivo: descrever as características obstétricas das gestantes submetidas à cesariana segundo a Classificação de Robson em um hospital universitário. Método: descritivo, retrospectivo, com abordagem quantitativa, com dados secundários de 294 prontuários de gestantes submetidas à cesariana no Hospital Universitário da Universidade Federal do Maranhão, no período de janeiro a dezembro de 2015. Resultados: predominância de nulíparas $(51,02 \%)$, submetidas à cesárea antes do início do trabalho de parto $(57,15 \%)$, com gestação a termo $(37,76 \%)$, com feto único $(96,3 \%)$, em apresentação cefálica $(90,82 \%)$. 0 grupo da Classificação de Robson com maior prevalência $(28,23 \%)$, foi o grupo 5 (multíparas com pelo menos uma cesárea anterior, feto único, cefálico, $\geq 37$ semanas). Conclusão: o hospital apresenta uma alta taxa de cesariana, totalizando 49,3\%, mesmo se tratando de um estudo realizado em uma maternidade referência para gestantes de alto risco. A pesquisa permitiu conhecer o perfil sociodemográfico e as características obstétricas das usuárias, dados importantes para o planejamento da assistência.
\end{abstract}

Descritores: Cesárea; parto obstétrico; classificação; gravidez.

\section{ABSTRACT}

Objective: to describe obstetric characteristics of pregnant women undergoing cesarean section at a University Hospital, by the Robson classification. Method: this quantitative, retrospective, descriptive study used secondary data from 294 medical records of pregnant women undergoing cesarean section at the hospital of Maranhão Federal University, from January to December 2015. Results: participants were predominantly nulliparous (51.02\%), underwent caesarean section before onset of labor $(57.15 \%)$, with term pregnancies $(37.76 \%)$ and a single fetus $(96.30 \%)$ in vertex presentation $(90.82 \%)$. Robson Classification group 5 (multiparous with at least one previous cesarean section, single fetus, cephalic, $\geq 37$ weeks ) was the most prevalent (28.23\%). Conclusion: the caesarean section rate at this hospital is high $(49.3 \%)$, even for a study at a referral maternity facility for high-risk pregnancies. The study revealed the users' sociodemographic profile and obstetric characteristics, which are important information for planning care.

Descriptors: Cesarean section; obstetric delivery; classification; pregnancy.

\section{RESUMEN}

Objetivo: describir las características obstétricas de las mujeres embarazadas sometidas a cesárea según la clasificación de Robson en un hospital universitario. Método: descriptivo, retrospectivo, con abordaje cuantitativo, con datos secundarios de 294 registros médicos de mujeres embarazadas sometidas a cesárea en el Hospital Universitario de la Universidad Federal de Maranhão, en el período de enero a diciembre de 2015. Resultados: predominio de nulíparas $(51,02 \%)$ que se sometieron a cesárea antes del comienzo del trabajo de parto $(57,15 \%)$, con embarazos a término $(37,76 \%)$ con un solo feto $(96,3 \%)$ en presentación cefálica de vértice $(90,82 \%)$. El grupo de la Clasificación de Robson con mayor prevalencia $(28,23 \%)$ fue el grupo 5 (multíparas con al menos una cesárea anterior, feto único, cefálico, $\geq 37$ semanas). Conclusión: el hospital tiene una alta tasa de cesáreas, un total de $49,3 \%$, incluso cuando se trata de un estudio realizado en un centro de maternidad de referencia mujeres embarazadas de alto riesgo. La investigación permitió conocer el perfil sociodemográfico y las características obstétricas de las pacientes, datos importantes para la planificación de la asistencia.

Descriptores: Cesárea; parto obstétrico; clasificación; embarazo.

\section{INTRODUÇÃO}

A gestação é um evento fisiológico que envolve mudanças em vários aspectos: físico, social e emocional e deve ser vivida pelas gestantes e profissionais de saúde como uma experiência de vida saudável. Por outro lado, trata-se de uma situação delicada que pode implicar riscos tanto para a mãe, quanto para o feto e há um grupo de gestantes que, por características inerentes, pode apresentar maiores chances de evolução desfavorável, são as chamadas gestantes de alto risco ${ }^{1}$.

'Enfermeira. Especialização. Aluna do Curso de Residência Multiprofissional em Saúde da Mulher. Universidade Federal do Maranhão. São Luís, Brasil. E-mail: liendne@hotmail.com

"Enfermeiro. Mestre. Preceptor do Curso de Residência Multiprofissional em Saúde da Mulher. Universidade Federal do Maranhão. São Luís, Brasil. E-mail: rivaldolirafilho@gmail.com

'"'Enfermeira. Mestre. Preceptora do Curso de Residência Multiprofissional em Saúde da Mulher. Universidade Federal do Maranhão. São Luís, Brasil. E-mail: roselustosa@hotmail.com 
Ao longo dos anos a Organização Mundial da Saúde (OMS) vem em um movimento constante para construção de um novo paradigma de atenção à saúde da mulher no ciclo gravídico puerperal. Desta maneira, busca-se o atendimento obstétrico integral permeado por uma assistência de qualidade, pautada em práticas assistenciais baseadas em evidências científicas, no direito de escolhas das mulheres e minimização de intervenções neste momento ${ }^{2}$, assim é a escolha da via parto, que apesar de respeitar a opinião e o direito da mulher de escolher como parir, deverá ser pautada em evidências científicas.

A escolha da via do parto pela mulher, todavia, poderá gerar nelas opiniões de soluções que mais satisfaçam seus anseios e expectativas, de forma independente das condições clínicas, obstétricas e orientações propiciadas em sua assistência pré-natal. A opinião feminina refletindo sua autonomia, de certa forma, deverá estar acoplada, e não sobreposta aos critérios clinicamente conhecidos. As institucionalizações da assistência ao parto e os avanços tecnológicos vêm alcançando maiores benefícios maternos e fetais, reduzindo a morbiletalidade do ciclo grávido puerperal; contudo, ainda se observa nesses procedimentos a prática de inúmeras intervenções desnecessárias. A alta incidência de partos cesáreos sem indicações precisas é motivo de preocupação mundial. O procedimento associa-se a taxas mais elevadas de mortalidade materna, quatro a cinco vezes maiores em relação aos partos vaginais e, ainda, encontra-se associado a morbidade e mortalidade perinatais ${ }^{1}$.

A atenção obstétrica no Brasil ainda está focada no modelo biomédico, o que tem contribuído para o aumento de procedimentos invasivos e intervencionistas durante o trabalho de parto, e consequentemente, refletindo nos altos índices de morbimortalidade materna e perinatal ${ }^{3}$. A escolha da via de parto pela equipe médica é uma decisão que deve ser tomada de acordo com cada caso e é essencial o esclarecimento da gestante e sua família, com informações precisas e de uma maneira que lhes seja compreensível, quanto às opções existentes e os riscos de cada uma delas, garantindo a participação da gestante no processo de decisão. Cabe ressaltar que gravidez de risco não é sinônimo de cesariana. Em alguns casos é possível a indução do parto vaginal, ou mesmo aguardar o seu início espontâneo ${ }^{1}$.

Em condições ideais, a operação cesariana é uma cirurgia segura e com baixa frequência de complicações graves. Além disso, quando realizada em decorrência de razões médicas, a operação cesariana é efetiva na redução da mortalidade materna e perinatal. Entretanto, é frequentemente utilizada de forma desnecessária, sem razões médicas que possam justificar as altas taxas observadas no Brasil ${ }^{4}$. O parto por via vaginal é mais seguro, tanto para a mãe quanto para o bebê, pois a morbidade materna é frequente e mais grave após a cesariana ${ }^{5}$.

As cesáreas sem indicação médica estão associadas a um maior risco de infecção puerperal, mortalidade e morbidade materna, prematuridade, mortalidade neonatal e consequente elevação de gastos para o sistema de saúde. A busca por fatores que justifiquem esses aumentos é essencial para que soluções possam ser pensadas ${ }^{1}$.

Para tanto, como forma de avaliar e monitorar a taxa de cesárea do hospital em estudo, a pesquisa teve como objetivo verificar as características obstétricas das gestantes submetidas à cesariana segundo a Classificação de Robson.

\section{REVISÃO DE LITERATURA}

Em 1985, a comunidade médica internacional declarou que a taxa ideal de cesárea seria entre $10 \%$ e $15 \%$. No entanto, as cesarianas têm aumentado tanto nos países desenvolvidos, como naqueles em desenvolvimento ${ }^{6}$. Esse aumento do número de cesarianas tem sido analisado e discutido em todo mundo e se tornado uma questão de saúde pública, já que, quando utilizada de forma desnecessária, pode representar risco adicional para o binômio mãe-bebê7.

No Brasil a situação não é diferente. Em muitos hospitais, o parto abdominal tornou-se a norma: o Brasil é hoje um dos países com maiores taxas de cesárea em todo o mundo. Mais do que apenas uma taxa sobre a via de parto, a alta taxa de cesáreas indica o grau de hipermedicalização da maternidade em que se situa o país ${ }^{8}$.

Segundo a OMS, ainda não existe uma classificação internacional que seja utilizada para facilitar a identificação das taxas de cesarianas, de forma significativa, nas maternidades de várias cidades ou regiões. No entanto, algumas características obstétricas vêm sendo estudadas com o objetivo de avaliar as indicações de cesarianas. O conhecimento e a identificação adequada dos fatores de risco para cesariana são de fundamental importância para planejar ações de saúde voltadas à prevenção de morbimortalidade materna-infantilí.

Proposta pelo médico Michael Robson, em 2001, a classificação dos 10 grupos (também conhecida como Classificação de Robson) tem sido bastante utilizada nos últimos anos. Essa classificação agrupa as gestantes de acordo com suas características obstétricas ${ }^{6}$.

Esse sistema classifica todas gestantes em um dentre 10 grupos que são mutuamente exclusivos e totalmente inclusivos. Os grupos são criados baseando-se em cinco parâmetros que são colhidos rotineiramente em todas as maternidades, o que permite uma comparação entre as taxas de cesáreas sem muitos fatores de confusão: paridade (nulípara ou multípara com e sem cesárea anterior); início do parto (espontâneo, induzido ou cesárea antes do início do 
trabalho de parto); idade gestacional (pré-termo ou a termo); apresentação/situação fetal (cefálica, pélvica ou transversa) e número de fetos (único ou múltiplo).

A classificação é simples, robusta, reproduzível, clinicamente relevante, e prospectiva - o que significa que todas as gestantes internadas para o parto podem ser imediatamente classificadas em um dos 10 grupos, usando apenas algumas dessas características básicas. A classificação permite a comparação e a análise das taxas de cesáreas dentro e entre esses grupos 6 .

A OMS propõe que a Classificação de Robson seja aplicada como instrumento padrão em todo o mundo para avaliar, monitorar e comparar taxas de cesáreas ao longo do tempo em um mesmo hospital e entre diferentes hospitais ${ }^{6}$.

\section{METODOLOGIA}

Trata-se de uma pesquisa de campo, descritiva, retrospectiva, de abordagem quantitativa, com dados secundários obtidos junto aos prontuários de gestantes submetidas ao parto cesáreo em 2015. Realizada no Serviço de Arquivo Médico (SAME) do Hospital Universitário da Universidade Federal do Maranhão (HUUFMA) - Unidade Materno Infantil e Unidade Presidente Dutra. Trata-se de uma pesquisa no Hospital-Maternidade, de nível terciário, referência para gestações de alto risco. A coleta de dados se deu em dezembro de 2016 a fevereiro de 2017.

Foram excluídas aquelas pacientes cujas informações de identificação não estavam legíveis e os prontuários não foram localizados, assim como aquelas que tiveram como produto do nascimento um feto morto (aquele que nasce pesando mais de $500 \mathrm{~g}$ e que não tem evidência de vida após o nascimento).

Solicitou-se o livro de registros dos partos, realizados no ano de 2015, no Centro Cirúrgico Obstétrico, onde consta o número do prontuário, nome, procedimento realizado e data. Solicitou-se ao SAME a disponibilização dos prontuários para rastreamento e coleta de dados.

Nesse período ocorreram 3454 partos, sendo 1750 (50,7\%) normais e 1704 (49,3\%) cesarianas. Dos 1704 partos cesáreas, foi definida uma amostra de 314 prontuários (18,4\%), que foram selecionados de forma sistemática (escolhidos de 5 em 5). Estes 314 prontuários representam 18,4\% dos partos cesáreas, para um erro amostral de 5\%, para um intervalo de confiança de $95 \%$, $(p<0,05)$.

Todos os prontuários da amostra foram de cesarianas de fetos vivos, visto, que de acordo com os critérios de exclusão, os abortos e fetos mortos foram eliminados antes da coleta de dados. Dos 314 prontuários, 12 não foram localizados por incoerência dos números de identificação dos prontuários e oito foram perdidos por ausência de dados que comprometiam os resultados da pesquisa, resultando 294 prontuários na amostra.

A coleta dos dados foi feita por meio do preenchimento de um formulário online, as variáveis coletadas compreenderam características sociodemográficas e obstétricas, levando-se em consideração a Classificação da Escala de Robson ${ }^{6}$. Foram analisados dados sobre paridade (nulípara ou multípara com e sem cesárea anterior), início do parto (espontâneo, induzido ou cesárea antes do início do trabalho de parto), idade gestacional (pré-termo ou a termo), apresentação/situação fetal (cefálica, pélvica ou transversa) e número de fetos (único ou múltiplo).

As pacientes foram agrupadas em um dentre os 10 grupos da Classificação ${ }^{6}$ conforme as peculiaridades da gestação. Grupo 1 (nulíparas, feto único, cefálico, $\geq 37$ semanas, em trabalho de parto espontâneo); grupo 2 (nulíparas, feto único, cefálico, $\geq 37$ semanas, cujo parto é induzido ou que são submetidas à cesárea antes do início do trabalho de parto);grupo 3 (multíparas sem cesárea anterior, feto único, cefálico, $\geq 37$ semanas, em trabalho de parto espontâneo); grupo 4 (multíparas sem cesárea anterior, feto único, cefálico, $\geq 37$ semanas, cujo parto é induzido ou que são submetidas à cesárea antes do início do trabalho de parto);grupo 5 (todas multíparas com pelo menos uma cesárea anterior, feto único, cefálico, $\geq 37$ semanas); grupo 6 (todas nulíparas com feto único em apresentação pélvica). Prosseguindo, grupo 7 (todas multíparas com feto único em apresentação pélvica, incluindo aquelas com cesárea (s) anterior (es)); grupo 8 (todas mulheres com gestação múltipla, incluindo aquelas com cesárea (s) anterior (es)); grupo 9 (todas gestantes com feto em situação transversa ou oblíqua, incluindo aquelas com cesárea (s) anterior (es)); grupo 10 (todas gestantes com feto único e cefálico, < 37 semanas, incluindo aquelas com cesárea (s) anterior (es)).

Os dados foram tabulados e analisados através do programa Microsoft Office Excel 2010, sendo então organizados e as variáveis foram demonstradas em tabelas.

O projeto de pesquisa foi submetido à Comissão Científica e ao Comitê de Ética em Pesquisa do HUUFMA e aprovado com o no 1.872 .029 . 


\section{RESULTADOS E DISCUSSÃo}

Foram analisados 294 prontuários de gestantes submetidas à cesariana no ano de 2015 . As faixas etárias variaram entre menores de 15 anos até 35 anos ou mais, sendo, 135 (45,92\%) de 16 a 25 anos, 122 (41,50\%) de 26 a 34 anos e 33 $(11,22 \%)$ com 35 anos ou mais. Quanto à situação conjugal, 118 (40,14\%) estavam em uma união estável, 106 (36,05\%) eram solteiras e 67 (22,79\%) casadas. Em relação à escolaridade, a maioria, 156 (53,06\%), possuía o nível médio completo ( $2 \circ$ grau) e 215 (73,13\%) se autodeclararam pardas, conforme mostra a Tabela 1.

TABELA 1: Caracterização sociodemográfica das gestantes submetidas à cesariana no ano de 2015. São Luís-MA, 2017. ( $N=294)$

\begin{tabular}{|c|c|c|}
\hline Variáveis & $f$ & $\%$ \\
\hline \multicolumn{3}{|l|}{ Faixa etária } \\
\hline Até 15 anos & 4 & 1,36 \\
\hline 16 a 25 anos & 135 & 45,92 \\
\hline 26 a 34 anos & 122 & 41,50 \\
\hline 35 anos ou mais & 33 & 11,22 \\
\hline \multicolumn{3}{|l|}{ Situação conjugal } \\
\hline Casada & 67 & 22,79 \\
\hline Solteira & 106 & 36,05 \\
\hline União estável & 118 & 40,14 \\
\hline Divorciada & 1 & 0,34 \\
\hline Sem informação & 2 & 0,68 \\
\hline \multicolumn{3}{|l|}{ Escolaridade } \\
\hline Não sabe ler/ escrever & 1 & 0,34 \\
\hline Nível fundamental completo (1o grau completo) & 16 & 5,45 \\
\hline Nível fundamental incompleto (1o grau incompleto) & 36 & 12,24 \\
\hline Nível médio completo (2ograu completo) & 156 & 53,06 \\
\hline Nível médio incompleto ( $2^{\circ}$ grau incompleto) & 41 & 13,95 \\
\hline Superior completo & 19 & 6,46 \\
\hline Superior incompleto & 19 & 6,46 \\
\hline Sem informação & 6 & 2,04 \\
\hline \multicolumn{3}{|l|}{ Raça } \\
\hline Amarela & 1 & 0,34 \\
\hline Branca & 43 & 14,63 \\
\hline Negra & 35 & 11,90 \\
\hline Parda & 215 & 73,13 \\
\hline
\end{tabular}

Em relação à paridade, 150 (51,02\%) das gestantes eram nulíparas, e 108 (36,74\%) eram multíparas com cesárea anterior. Em relação ao início do trabalho de parto, nota-se uma predominância do número de cesáreas realizadas antes do início do trabalho de parto - 168 (57,15\%). Já 83 (28,23\%) parturientes entraram em trabalho de parto espontâneo, mas por algum motivo foram encaminhadas para a cesariana. Considerando a idade gestacional, observa-se que 111 (37,76\%) partos foram a termo e 45 (15,30\%) prematuros. Em relação à apresentação/situação fetal, 267 (90,82\%) dos fetos apresentavam-se em posição cefálica, enquanto que 24 (8,16\%) em posição pélvica. Já em relação ao número fetos, $283(96,30 \%)$ das pacientes apresentavam gestação com feto único, como descrito na Tabela 2.

A partir das características obstétricas, ao distribuir as gestantes dentro dos 10 grupos da Classificação de Robson, nota-se que a maior ocorrência de cesárea, 83 (28,23\%), foi encontrada no grupo 5 (todas multíparas com pelo menos uma cesárea anterior, feto único, cefálico, $\geq 37$ semanas), seguida do grupo 2 (nulíparas, feto único, cefálico, $\geq 37$ semanas, cujo parto é induzido ou que são submetidas à cesárea antes do início do trabalho de parto) com 72 (24,49\%), como mostra a Tabela 3.

Ao compararmos o grupo 5 com grupos de gestantes com características semelhantes, no caso os grupos 3 (multíparas sem cesárea anterior, feto único, cefálico, $\geq 37$ semanas, em trabalho de parto espontâneo) e 4 (multíparas sem cesárea anterior, feto único, cefálico, $\geq 37$ semanas, cujo parto é induzido ou que são submetidas à cesárea antes do início do trabalho de parto), as taxas de cesárias foram menores, sendo 7 (2, 38\%) e 20 (6,80\%), respectivamente.

As taxas de cesarianas podem variar por diversos fatores, dentre eles, fatores socioeconômicos, culturais e obstétricos. De acordo com o Ministério da Saúde ${ }^{4}$, o Brasil vive uma epidemia de operações cesarianas, com aproximadamente 1,6 milhão de operações cesarianas realizadas a cada ano. Nas últimas décadas, a taxa nacional de 
operações cesarianas tem aumentado progressivamente, e a operação cesariana tornou-se o modo mais comum de nascimento no País. A taxa de operação cesariana no Brasil está ao redor de 56\%, havendo uma diferença significativa entre os serviços privados de saúde (85\%) e os serviços públicos de saúde (40\%), consideravelmente menor, mas ainda elevada, tomando-se por base a taxa preconizada pela OMS de até $15 \%^{9}$.

TABELA 2: Características obstétricas das gestantes submetidas à cesariana no ano de 2015, segundo a Classificação de Robson. São Luís-MA, 2017. $(\mathrm{N}=294)$

\begin{tabular}{|c|c|c|}
\hline Variáveis & f & $\%$ \\
\hline \multicolumn{3}{|l|}{ Paridade } \\
\hline Nulípara & 150 & 51,02 \\
\hline Multípara sem cesárea anterior & 36 & 12,24 \\
\hline Multípara com cesárea anterior & 108 & 36,74 \\
\hline \multicolumn{3}{|l|}{ Início do trabalho de parto } \\
\hline Espontâneo & 83 & 28,23 \\
\hline Induzido & 43 & 14,62 \\
\hline Cesárea antes do início do trabalho de parto & 168 & 57,15 \\
\hline \multicolumn{3}{|l|}{ Idade gestacional } \\
\hline$<37$ semanas & 45 & 15,30 \\
\hline 37 semanas a 38 semanas e 6 dias & 75 & 25,51 \\
\hline 39 semanas a 40 semanas e 6 dias & 111 & 37,76 \\
\hline 41 semanas a 41 semanas e 6 dias & 48 & 16,33 \\
\hline$>42$ semanas & 15 & 5,10 \\
\hline \multicolumn{3}{|l|}{ Apresentação/ Situação fetal } \\
\hline Cefálica & 267 & 90,82 \\
\hline Pélvica & 24 & 8,16 \\
\hline Transversa & 3 & 1,02 \\
\hline \multicolumn{3}{|l|}{ Número de fetos } \\
\hline Único & 283 & 96,3 \\
\hline Múltiplo & 11 & 3,7 \\
\hline
\end{tabular}

TABELA 3: Distribuição das gestantes submetidas à cesariana no ano de 2015, segundo a Classificação de Robson. São Luís-MA, 2017.

\begin{tabular}{|c|c|c|}
\hline Grupos de Robson & f & $\%$ \\
\hline Grupo 1 - Nulíparas, com feto único, cefálico, $\geq 37$ semanas, em trabalho de parto espontâneo & 42 & 14,29 \\
\hline $\begin{array}{l}\text { Grupo } 2 \text { - Nulíparas, com feto único, cefálico, } \geq 37 \text { semanas, cujo parto é induzido ou que são } \\
\text { submetidas à cesárea antes do início do trabalho de parto }\end{array}$ & 72 & 24,49 \\
\hline $\begin{array}{l}\text { Grupo } 3 \text { - multíparas sem cesárea anterior, com feto único, cefálico, } \geq 37 \text { semanas, em trabalho de } \\
\text { parto espontâneo }\end{array}$ & 7 & 2,38 \\
\hline $\begin{array}{l}\text { Grupo } 4 \text { - multíparas sem cesárea anterior, com feto único, cefálico, } \geq 37 \text { semanas, cujo parto é } \\
\text { induzido ou que são submetidas à cesárea antes do início do trabalho de parto }\end{array}$ & 20 & 6,80 \\
\hline $\begin{array}{l}\text { Grupo } 5 \text { - todas multíparas com pelo menos uma cesárea anterior, com feto único, cefálico, } \geq 37 \\
\text { semanas }\end{array}$ & 83 & 28,23 \\
\hline Grupo 6 - todas nulíparas com feto único em apresentação pélvica & 12 & 4,08 \\
\hline $\begin{array}{l}\text { Grupo } 7 \text { - todas multíparas com feto único em apresentação pélvica, incluindo aquelas com } \\
\text { cesárea(s) anterior(es) }\end{array}$ & 9 & 3,06 \\
\hline Grupo 8 - todas mulheres com gestação múltipla, incluindo aquelas com cesárea (s) anterior (es) & 11 & 3,74 \\
\hline $\begin{array}{l}\text { Grupo } 9 \text { - todas gestantes com feto em situação transversa ou oblíqua, incluindo aquelas com } \\
\text { cesárea(s) anterior(es) }\end{array}$ & 3 & 1,02 \\
\hline $\begin{array}{l}\text { Grupo } 10 \text { - todas gestantes com feto único e cefálico, < } 37 \text { semanas, incluindo aquelas com cesárea(s) } \\
\text { anterior(es) }\end{array}$ & 35 & 11,90 \\
\hline Total & 294 & 100,00 \\
\hline
\end{tabular}

Verificou-se maior frequência de gestantes na faixa etária que de 16 a 25 anos, e seguindo-se o grupo de 26 a 34 anos, resultados semelhantes ao da pesquisa sobre o perfil epidemiológico das parturientes submetidas à cesariana em Botucatu-SP ${ }^{10}$, onde a faixa etária variou de 19 a 36 anos. Embora não existam justificativas biológicas claras para tal, a hipótese é de que mulheres mais velhas estão sujeitas a ter mais comorbidades $^{11}$, o que poderia contribuir para a indicação de cesarianas. A idade materna é considerada como um fator gerador de risco para a gestação. Para o 
Ministério da Saúde, gestantes com idade igual ou superior a 35 anos são consideradas tardias ou em idade avançada, sendo mais suscetíveis a desenvolverem complicações durante a gravidez, o que torna a gestação de alto risco ${ }^{12}$.

Em relação à situação conjugal, a maior parte das gestantes estava em uma união estável, porém um número relativamente alto era solteira. Ainda no estudo em Botucatu-SP foi observado que a maioria era casada, seguida de solteira ${ }^{10}$. Quem já passou pela experiência da gestação ou mesmo acompanhou de perto, pode facilmente comprovar que independente da configuração familiar, este período traz à tona uma série de emoções e de decisões que impactam o cotidiano das já formadas ou futuras famílias, sendo assim, diversos países, que desenvolvem estudos e pesquisas aplicadas a esta temática, ressaltam a importância e os resultados positivos do engajamento ativo dos homens em todo esse processo de gestação, parto e puerpério. Estimular a participação do pai/parceiro durante todo esse processo pode ser fundamental para o bem estar biopsicossocial da mãe, do bebê e dele próprio ${ }^{13}$.

Quanto ao grau de instrução, a maioria das pacientes possuía o ensino médio completo, como encontrado no estudo que analisa a caracterização sociodemográfica e epidemiológica de cesarianas em uma maternidade pública de Teresina ${ }^{14}$. No entanto, esse dado tem mais significância quando a gestante é investigada quanto à preferência da via de parto, o que não é o caso deste estudo. As mulheres que utilizam o setor privado de saúde, que hipoteticamente teriam melhor acesso às informações e a serviços de qualidade, são as que mais se submetem à cirurgia ${ }^{15}$.

Houve uma prevalência de nulíparas submetidas à cesariana. Altas taxas de cesarianas em primíparas são particularmente preocupantes porque implicam alta probabilidade de futuras cesarianas, pois, na prática, uma cesariana prévia constitui indicação quase absoluta para nova cesariana ${ }^{16}$.

As multíparas com cesárea anterior, neste estudo, também contribuíram para o alto índice de cesariana. A primeira cesariana contribui com grande número de procedimentos na taxa global. O parto por via alta indicado por iteratividade, ou seja, pela presença de uma cicatriz uterina prévia ajuda, de forma muito expressiva, no aumento das taxas globais de cesarianas. Nos Estados Unidos, um terço de todas as cesarianas é realizado em pacientes com cesariana prévia ${ }^{17}$. Cesarianas prévias têm sido apontadas como fator de manutenção das altas taxas dessa cirurgia em países desenvolvidos. Reforçando tal questão, em estudo realizado, mulheres com parto vaginal prévio têm chance menor (em torno de 25 vezes) de optarem por cesariana. O parto vaginal após cesariana, quando comparado à cesariana de rotina indicada por iteratividade, apresenta resultados favoráveis ${ }^{18}$. Revisão sistemática com metanálise de 203 estudos demonstrou que a mortalidade materna aumentou significativamente com cesarianas de repetição, comparando-se esses dados com os resultados dos partos vaginais após cesariana ${ }^{19}$.

O alto índice de cesárea antes do início do trabalho de parto, as gestações a termo (39 semanas a 40 semanas e 6 dias) e as gestações a termo inicial (37 semanas a 38 semanas e 6 dias) foram as que mais prevaleceram, o que se contrapôs a outro estudo com um percentual elevado (30\%) das gestantes em idade pré-termo ${ }^{10}$. As altas prevalências de cesarianas antes do trabalho de parto e de termos precoces nascidos por cesarianas são preocupantes, pois cesarianas antes de 39 semanas aumentam o risco de morbidade neonatal ${ }^{20}$ e de desfechos negativos de longo prazo, como resultados mais baixos em testes de leitura e de matemática na infância, quando comparados com crianças nascidas após 39 semanas de gestação ${ }^{21}$.

Em mais de $90 \%$ das cesarianas ocorreu apresentação cefálica, o que colaboraria para que o parto vaginal se tornasse possível. Entretanto variações da apresentação cefálica podem ser indicativas de cesarianas quando, por exemplo, houver falha nas manobras de correção de distócias, sinais de parto obstruído ou de hipoxemia fetal ${ }^{22}$. Neste estudo, não foram registrados os motivos pelos quais a equipe obstétrica optou pelo parto por via alta, porém, é inegável uma incidência exagerada de partos cesáreos, o que leva a uma reflexão sobre a real necessidade deste procedimento.

Em relação ao número de fetos, embora a maioria das gestações submetidas à cesariana tenham sido de feto único, estudos recentes apontam que, no caso de gestações múltiplas, os resultados perinatais não são melhorados pelos partos por cesariana, quando o primeiro gêmeo se apresenta em posição cefálica. Assim, mulheres que tenham gêmeos, em apresentação cefálica/cefálica ou cefálica/ não cefálica, deverão ser aconselhadas a tentar o parto vaginal ${ }^{23}$.

Na maioria dos estudos em que a Classificação de Robson foi adotada, a cesárea foi mais frequente nos grupos 5 (todas multíparas com pelo menos uma cesárea anterior, feto único, cefálico, $\geq 37$ semanas) e 2 (nulíparas, feto único, cefálico, $\geq 37$ semanas, cujo parto é induzido ou que são submetidas à cesárea antes do início do trabalho de parto) ${ }^{24,25}$, assim como neste estudo. O grupo 5 é composto por multíparas com pelo menos uma cesárea anterior, o que reforça que a cesárea prévia eleva a indicação de parto operatório em gestações futuras. Por outro lado, ao comparar o grupo 5 com outros grupos similares de mulheres (multíparas), porém sem cicatriz uterina anterior, como os grupos 3 (multíparas sem cesárea anterior, feto único, cefálico, $\geq 37$ semanas, em trabalho de parto espontâneo) e 4 (multíparas sem cesárea anterior, feto único, cefálico, $\geq 37$ semanas, cujo parto é induzido ou que são submetidas à cesárea antes do início do trabalho de parto), pode-se inferir que ter tido parto vaginal anterior diminui as chances de parto por cesárea em gestações subsequentes ${ }^{25}$. 
Além disso, houve uma alta taxa de cesárea em mulheres sem cicatriz uterina prévia, com trabalho de parto induzido ou cesárea antes do início do trabalho de parto (grupo 2), mostrando a necessidade de se rever as atuais indicações e formas de indução do trabalho de parto, assim como as reais indicações de cesárea nessas mulheres ${ }^{25}$.

\section{CONCLUSÃO}

Conclui-se por meio deste estudo que o hospital apresenta uma alta taxa de cesariana, mesmo se tratando de um estudo realizado em uma maternidade referência para gestantes de alto risco. A maioria das pacientes submetidas à cesariana era nulípara, seguindo-se as de multíparas com cesárea anterior, o que reforça a importância da prevenção de cesárea em primigestas, a fim de evitar indicações em gestações futuras.

Ressalta-se também a importância do uso da Classificação de Robson, como instrumento para conhecimento do perfil das usuárias através das características obstétricas, além de colaborar para o acompanhamento, planejamento e tomada de decisões da equipe.

As limitações do presente estudo são relacionadas à escassez de material publicado sobre o tema direcionado à Classificação de Robson, às incoerências de números de certos prontuários e à qualidade das informações nos mesmos.

Destacam-se a relevância desta temática e a necessidade de outras pesquisas para esclarecer os distintos fatores que influenciam a escolha da via de parto, no sentido de reduzir as taxas de cesarianas e promover uma assistência de qualidade, com o mínimo de intervenções possíveis no binômio mãe-bebê.

\section{REFERÊNCIAS}

1. Ministério da Saúde (Br). Secretaria de Atenção à Saúde. Departamento de Ações Programáticas Estratégicas. Gestação de alto risco: manual técnico. Brasília (DF): Ministério da Saúde; 2010.

2. Pinto LMTR, Trezzall MCSF, Santos AAP, Melo GC, Silva JMO, Oliveira LLF. Food management during childbirth under woman's perception.Rev. enferm. UERJ [Internet]. 2017 [cited 2018 Jan 20]; 25:e14205. DOI: https://doi.org/10.12957/reuerj.2017.14205

3. Andrade LFB, Rodrigues QP, Silva R CV. Good Partices in obstetric care and its interface with humanization of assistance. Rev enferm UERJ [Internet]. 2017 Dez [cited 2018 Jan 20]; 25:e26442. DOI: http://dx.doi.org/10.12957/reuerj.2017.26442

4. Ministério da Saúde (Br). Secretaria de Ciência, Tecnologia e Insumos Estratégicos. Diretrizes de Atenção à Gestante: a operação cesariana: protocolo. Brasília (DF): Ministério da Saúde; 2016.

5. Reis SLS, Penteado CEM, Chatkin MN, Estrela MS, Porto PG, Munaretto MM. Parto normal X parto cesáreo: análise epidemiológica em duas maternidades no sul do Brasil. Rev. AMRIGS. 2009; 53(1):7-10.

6. World Health Organization. WHO statement on caesarean section rates. Geneva (Swi): WHO; 2015.

7. Torloni MR, Betran AP, Souza JP, Widmer M, Allen T, Gulmezoglu M, et al. Classifications for cesarean section: a systematic review. PLoS ONE. [Internet] 2011 [cited 2018 Jan 20]; 6(1): e14566. DOI: http://doi.org/10.1371/journal.pone.0014566

8. Souza JP, Pileggi-Castro C. On labor and childbirth: the importance of quaternary prevention. Cad. Saúde Pública (Online). 2014 [cited 2018 Jan 20]; 30 (supl 1):S11-3. DOI: http://dx.doi.org/10.1590/0102-311XPE02S114

9. Sakae TM, Freitas PF, d'Orsi E. Factors associated with cesarean section rates in a university hospital. Rev. saúde pública. (Online) 2009 [cited 2018 Jan 20]; 43(3): 472-80. DOI: http://dx.doi.org/10.1590/S0034-89102009000300011

10. Neves APA. Perfil epidemiológico de parturientes submetidas à cesariana na maternidade de um hospital escola de Botucatu-SP [monografia de graduação]. Botucatu (SP): Universidade Estadual Paulista; [Internet] 2011 [cited 2018 Jan 20]; Available from: https://repositorio.unesp.br/bitstream/handle/11449/120175/neves_apa_tcc_botfm.pdf?sequence=1\&isAllowed=y

11. Gama AS, Giffin KM, Tuesta AA, Barbosa GP, D’Orsi E. Women's representations and experiences with vaginal and cesarean delivery in public and private maternity hospitals. Cad Saúde Pública (Online). 2009 [cited 2018 Jan 20]; 25: 2480-88. http://dx.doi.org/10.1590/S0102-311X2009001100017

12. Ministério da Saúde (Br). Secretaria de Atenção à Saúde. Departamento de Ações Programáticas Estratégicas. Gestação de alto risco: manual técnico. Brasília (DF): Ministério da Saúde; 2012.

13. Ministério da Saúde (Br). Secretaria de Atenção à Saúde. Departamento de Ações Programáticas Estratégicas. Guia do Pré-Natal do Parceiro para Profissionais de Saúde. Brasília (DF): Ministério da Saúde; 2016.

14. Ribeiro JF, Costa FS, Silva KR, Carvalho TES. Caracterização sociodemográfica e epidemiológica de cesarianas em uma maternidade pública de referência em Teresina. Rev. eletrônica gest. saúde. 2014 [cited 2018 Jan 20]; 3(5): 977-91. http://dx.doi.org/10.1590/0034-7167-2017-0325

15. Riscado LC, Jannotti CB, Barbosa, RHS. A decisão pela via de parto no Brasil: temas e tendências na produção da saúde coletiva. Texto contexto enferm. 2016 [cited 2018 Jan 20]; 25(1): e3570014. DOI: http://dx.doi.org/10.1590/01040707201600003570014

16. Iorra MRK, Namba A, Spillere RG, Nader SS, Nader PJH. Aspectos relacionados à preferência pela via de parto em um hospital universitário. Rev. AMRIGS. 2011; 55(3): 260-8.

17. Marshall NE, Fu R, Guise JM. Impact of multiple cesarean deliveries on maternal morbidity: a systematic review. Am. J. Obstet. Gynecol. [Internet]. 2011 [cited 2018 Jan 20]; 205(3): 262.e1-8. DOI: https://dx.doi.org/10.1016/j.ajog.2011.06.035

18. Faisal-Cury A, Menezes PR. Factors associated with preference for cesarean delivery. Rev. saúde pública (Online). 2006 [cited 2018 Jan 20]; 40(2): 226-32. DOI: http://dx.doi.org/10.1590/S0034-89102006000200007 
19. Guise JM, Denman MA, Emeis C, Marshall N, Walker M, Fu R, et al. Vaginal birth after cesarean: new insights on maternal and neonatal outcomes. Obstet. Gynecol. 2010 [cited 2018 Jan 20]; 115(6): 1267-78. DOI: https://doi.org/10.1097/AOG.0b013e3181df925f .

20. Hansen AK, Wisborg K, Uldbjerg N, Henriksen TB. Risk of respiratory morbidity in term infants delivered by elective caesarean section: cohort study. BMJ. 2008 [cited 2018 Jan 20]; 336(7635): 85-7. doi: https://doi.org/10.1136/bmj.39405.539282.BE

21. Noble KG, Fifer WP, Rauh VA, Nomura Y, Andrews HF. Academic achievement varies with gestational age among children born at term. Pediatrics. 2012 [cited 2018 Jan 20]; 130(2): e257-64. DOI: http://dx.doi.org/10.1542/peds.2011-2157

22. Haddad SEMT, Cecatti JG. Strategies directed to professionals for reducing unnecessary cesarean sections in Brazil. Rev. Bras. Ginecol Obstet. 2011 [cited 2018 Jan 20]; 33(5):252-62. DOI: http://dx.doi.org/10.1590/S0100-72032011000500008

23. Obstetric Care Consensus no 5: severe maternal morbidity: screening and review. Obstetric Care Consensus № 5 . Obstet Gynecol 2016 [cited 2018 Jan 20]; 128(3): e54-60. DOI: https://doi.org/10.1097/AOG.0000000000001642

24. Kelly S, Sprague A, Fell DB, Murphy P, Aelicks N, Guo Y, et al. Examining caesarean section rates in Canada using the Robson classification system. J. Obstet .Gynaecol .Can. 2013 [cited 2018 Jan 20]; 35(3): 06-14. DOI: https://doi.org/10.1016/S17012163(15)30992-0

25. Lee YY, Roberts CL, Patterson JA, Simpson JM, Nicholl MC, Morris JM, et al. Unexplained variation in hospital caesarean section rates. Med. J. Aust. [Internet]. 2013 [cited 2018 Jan 20]; 199(5):348-53. Available from:

https://www.ncbi.nlm.nih.gov/pubmed/23992192 